\title{
ARTÍCULO ORIGINAL \\ Sistemas de activación y de inhibición de conducta y su relación con el funcionamiento ejecutivo en excombatientes irregulares del conflicto armado colombiano
}

Fecha de recibido:

24 de marzo de 2020

Fecha de aprobación:

12 de mayo de 2020

Forma de citar este artículo: Montoya DA, Pareja AM, Valencia AM, Díaz CM, Trujillo N, Pineda DA. Sistemas de activación y de inhibición de conducta y su relación con funcionamiento ejecutivo en excombatientes irregulares del conflicto armado colombiano. Med UPB. 2020;39(2):2-10. DOI:10.18566/medupb.v39n2.a02

1 Universidad Pontificia Bolivariana, Escuela de Ciencias Sociales, Facultad de Psicología, Grupo de Investigación ECCO. Medellín, Colombia.

2 Grupo de Neuropsicología y Conducta UDEA-USB. Medellín, Colombia.

3 Grupo de Neuropsicología y Conducta (GRUNECO), Universidad de San Buenaventura, Medellín Colombia.

4 Escuela de Graduados, Universidad CES. Becaria Colciencias, convocatoria 757 de 2016. Medellín, Colombia.

5 Facultad Nacional de Salud Pública, Universidad de Antioquia (UDEA). Medellín, Colombia.

Dirección de correspondencia: David Andrés Montoya Árenas. Correo electrónico: david.neuropsi@gmail.com
Behavior activation and inhibition systems and their correlation with executive functioning in ex-combatants of the Colombian armed conflicto / Sistemas de ativação e de inibição de conduta e sua relação com o funcionamento executivo em ex-combatentes irregulares do conflito armado colombiano

David Andrés Montoya Arenas ${ }^{1,2}$, Ángela María Pareja ${ }^{3}$, Ana María Valencia ${ }^{3}$, Cecilia María Díaz Soto ${ }^{4}$, Natalia Trujillo Orrego ${ }^{5}$, David Antonio Pineda Salazar ${ }^{3}$

\section{RESUMEN}

Objetivo: Explorar la relación entre los sistemas de activación de conducta (SAC) / sistema de inhibición de conducta (SIC) y funciones ejecutivas (FE) en excombatientes del conflicto armado colombiano.

Metodología: Estudio observacional, exploratorio, con muestra no aleatoria de 39 excombatientes del programa de la Alta Consejería de la Presidencia para la Reintegración, residentes en Antioquia-Colombia, edad $35 \pm 7.6$ años, $87.2 \%$ hombres, y escolaridad $10 \pm 2.8$ grados; comparados con un grupo control de 14 no combatientes (edad $33 \pm 6.1$ años, $71.4 \%$ hombres y escolaridad $10 \pm 2.7$ grados). Se les administró un cuestionario de SAC/SIC, usando la sensibilidad a la recompensa (SR) y la sensibilidad al castigo (SC). Para la FE se aplicó la prueba Ineco Frontal Screening (IFS).

Resultados: Hubo alteraciones específicas de la FE: en la ejecución de instrucciones conflictivas (IC), memoria de trabajo verbal (MTV) y control inhibitorio verbal (CIV). Se evidenciaron correlaciones directas, con coeficientes de moderados a altos entre el SAC (SR) y la capacidad de abstracción CA, el control motor inhibitorio (CMI), la MTV, la memoria de trabajo espacial (MTE) y el funcionamiento ejecutivo global (IFSTOT). Conclusiones: Los excombatientes presentaron un rendimiento inferior en la memoria de trabajo verbal y el control inhibitorio verbal, lo que impacta las capacidades de anticipación, establecimiento de metas, diseño de planes, iniciación de conductas voluntarias y el control inhibitorio. Lo anterior puede generar la toma de decisiones equivocadas. Palabras clave: función ejecutiva; actividades cotidianas; conflictos armados; neuropsiquiatría.

\section{ABSTRACT}

Objective: To explore the correlations between behavior activation and inhibition systems (BAS/ BIS) and executive functions in ex-combatants of the Colombian armed conflict. Methodology: Observational, exploratory study, with a sample of 39 ex-combatants belonging to the "High Counseling of the Presidency for Reintegration" program, residents in Antioquia-Colombia, with an average of $35 \pm 7.6$ year of age, $87.2 \%$ were males, with school grades of $10 \pm 2.8$; who were compared with a control group of 14 non-combatants, with ages between $33 \pm 6.1$ years-old, $71.4 \%$ were males, and with school grades $10 \pm 2.7$. The BAS/ BIS questionnaire was administered in order to score the Reward Sensitivity (RS) and Punishment Sensitivity (PS). The EF was evaluated with INECO-Frontal Screening (IFS). Results: There were specific alterations of $\mathrm{EF}$ in the execution of conflicting instructions 
$(\mathrm{Cl})$, verbal working memory (MTV) and verbal inhibitory control (CIV). Direct correlations, moderate to high coefficients between SAC (SR) and CA abstraction capacity, CMI inhibitory motor control, MTV, spatial working memory (MTE), and global executive functioning (IFSTOT).

Conclusions: Colombian ex-combatants have lower performance in verbal working memory and verbal inhibitory control, which would affect anticipation, goal setting, plan design, initiation of voluntary behaviors and inhibitory control, which generates generation of wrong decisions.

Keywords: executive function; activities of daily living; armed conflicts; neuropsychiatry

\section{RESUMO}

Objetivo: Explorar a relação entre os sistemas de ativação de conduta (SAC) / sistema de inibição de conduta (SIC) e funções executivas (FE) em ex-combatentes do conflito armado colombiano.

Metodologia: Estudo observacional, exploratório, com amostra não aleatória de 39 ex-combatentes do programa da Alto Conselho da Presidência para a Reintegração, residentes em Antioquia-Colômbia, idade $35 \pm 7.6$ anos, $87.2 \%$ homens, e escolaridade $10 \pm 2.8$ graus; comparados com um grupo controle de 14 não combatentes (idade $33 \pm 6.1$ anos, $71.4 \%$ homens e escolaridade $10 \pm 2.7$ graus). Se Ihes administrou um questionário de SAC/SIC, usando a sensibilidade à recompensa (SR) e a sensibilidade ao castigo (SC). Para a FE se aplicou a prova Ineco Frontal Screening (IFS).

Resultados: Houve alterações específicas da FE: na execução de instruções conflitivas (IC), memória de trabalho verbal (MTV) e controle inibitório verbal (CIV). Se evidenciaram correlações diretas, com coeficientes de moderados a altos entre o SAC (SR) e a capacidade de abstração $\mathrm{CA}$, o controle motor inibitório (CMI), a MTV, a memória de trabalho espacial (MTE) e o funcionamento executivo global (IFSTOT).

Conclusões: Os ex-combatientes apresentaram um rendimento inferior na memória de trabalho verbal e o controle inibitório verbal, o que impacta as capacidades de antecipação, estabelecimento de metas, desenho de planos, iniciação de condutas voluntárias e o controle inibitório. $O$ anterior pode gerar a toma de decisões equivocadas.

Palavras chave: função executiva; atividades cotidianas; conflitos armados; neuropsiquiatria.

\section{INTRODUCCIÓN}

El sistema de inhibición de la conducta (SIC) [del inglés: Behavioral Inbibition System (BIS)] y el sistema de activación de la conducta [del inglés: Behavioral $A c^{-}$ tivation System (BAS)] hace referencia a factores de regulación interna de los comportamientos, a través de las emociones y de la cognición ${ }^{1,2}$.

Este modelo implica que el SAC influye sobre la sensibilidad para controlar los comportamientos a partir de la experiencia emocional activada por la gratificación, la recompensa o la motivación (por lo que también recibe el nombre de sensibilidad a la recompensa o SR). El SIC, en cambio, se activaría por experiencias emocionales aversivas relacionadas con la conducta, por eso también se denomina sensibilidad al castigo (SC).

De acuerdo con estudios en animales, el SAC (SR) se ha relacionado con circuitos prefrontales mediales y sus conexiones con el núcleo accumbens, predominantemente de tipo dopaminérgico. El SIC (SC) se implica circuitos monoaminérgicos del tallo cerebral y de la región medial de los lóbulos temporales, especialmente el núcleo amigdalino y el hipocampo ${ }^{1,2}$.

Estudios en humanos han relacionado el SAC (SR) con la búsqueda de actividades placenteras y de riesgos, lo que conduciría a trastornos externalizantes (abuso y dependencia de substancias, trastorno de atención e hiperactividad, trastornos del control de los impulsos, trastornos de la conducta) y con el uso preferencial de la 
agresión y la violencia ${ }^{3-7}$, mientras que el SIC (SC) se asocia con trastornos internalizantes, como la ansiedad y las alteraciones del estado de ánimo ${ }^{1,8}$.

La relación entre ambos sistemas y la actividad de los lóbulos frontales en humanos se ha establecido mediante estudios neurofisiológicos con electroencefalografía cuantitativa en reposo ${ }^{9,10}$, o con potenciales relacionados con eventos (paradigmas de inhibición de respuestas dominantes e impulsivas) ${ }^{9}$.

El SAC se ha estudiado en pacientes con trastorno afectivo bipolar (TAB) y en familiares de primer grado, usando resonancia magnética nuclear funcional (RMNf) con regiones de interés (ROI) focalizadas en los lóbulos frontales (Kollmann B, Scholz, Linke, Kirsch, Wessa, 2017). En cuanto al estudio de estos sistemas en otros tipos de pacientes, trabajos sobre conectividad cerebral con RMNf describieron la relación entre la búsqueda de recompensa y el consumo de sustancias ${ }^{11}$. En otro estudio se relacionaron los cambios en la SR y la SC con la impulsividad y las alteraciones ejecutivas en pacientes con enfermedad de Huntington ${ }^{12}$. Sin embargo, no se ha establecido la relación entre SAC/ SIC (SR-SC) y las funciones ejecutivas $(\mathrm{FE})$ en personas expuestas a combates en conflictos armados.

Por otra parte, se ha propuesto que las deficiencias en las FE disminuyen las habilidades para la resolución de problemas, así como la adaptación a ambientes sociales y la ejecución de respuestas adecuadas para evitar la agresión ${ }^{13}$; de ahí que las dificultades neuropsicológicas reducen el número de opciones que un individuo tiene para responder a cualquier situación ${ }^{14}$, en esta línea, la agresión sería probablemente producto de la desinhibición, en personas con déficits específicos en las $\mathrm{FE}^{15}$.

Es por esto que las investigaciones han mostrado cómo las alteraciones en la regulación de la conducta, la emoción y cognición, y fundamentalmente, procesos involucrados en la función ejecutiva, tiene que ver con la conducta antisocial y el abuso de drogas ${ }^{16,17}$. En cuanto a la conducta violenta, aunque los factores biológicos e individuales explican parte del problema, hay factores familiares, culturales y sociales que interactúan para crear una situación que favorece su aparición. De ahí que investigadores de diversas áreas del conocimiento busquen explicar los orígenes de este comportamiento ${ }^{18}$.

Para la comprensión de la naturaleza humana, y su forma de responder frente al ambiente, es fundamental el estudio de elementos neuropsicológicos asociados con la regulación de la conducta agresiva, especialmente en individuos que han estado involucrados en conflictos armados como el colombiano. El estudio de estos elementos implica aproximaciones multinivel, tal como lo plantean las neurociencias sociales cognitivas ${ }^{19}$, lo que permite identificar en esta población el funcionamiento ejecutivo asociado a la actividad cerebral de los lóbulos frontales reguladores de la cognición, conducta y emoción, y su relación con características de personalidad particulares que hacen que enfrenten situaciones conflictivas de una manera específica.

Por su parte, las FE se han descrito como la dimensión de más alto nivel de la cognición humana ("el cerebro del cerebro" ${ }^{20}$ y están compuestas por habilidades metacognitivas, emocionales y motivacionales que dirigen la conducta y estructuran el pensamiento. Se evalúan mediante tareas que comprenden la formulación de metas, la planificación de procesos, las estrategias para lograr los objetivos, y las aptitudes para llevar a cabo esas actividades $^{21,22}$. Si bien la neuropsicología y las neurociencias cognitivas han desarrollado instrumentos para la valoración de la metacognición, aún es insuficiente lo que se ha avanzado para dar cuenta del funcionamiento emocional y motivacional.

Las escasas investigaciones sobre las características comportamentales y neuropsicológicas, especialmente en FE, en actores del conflicto armado, como los excombatientes, dan cuenta de la necesidad de estudiar la relación entre los sistemas de inhibición y activación conductual y el funcionamiento ejecutivo en una población que ha manifestado conductas agresivas y violentas a lo largo de la vida. El propósito de este estudio exploratorio fue establecer las relaciones entre los SAC/ SIC (SR-SC) y las funciones ejecutivas medidas con una prueba de rastreo, en excombatientes del conflicto armado colombiano.

\section{METOdOLOGÍA}

La muestra de este estudio fue seleccionada por conveniencia. Estuvo compuesta por 39 excombatientes activos en el programa de la Alta Consejería de la Presidencia para la Reintegración (ACR) residentes en Antioquia, que cumplieran los criterios de inclusión y 14 controles homogéneos de ambos sexos; 10 (71.4\%) hombres y 4 (28.6\%) mujeres, con una edad promedio de 32 años y una escolaridad promedio de 10 años. La capacidad intelectual (CI) estimada de los controles fue significativamente superior a la de los combatientes. No hubo otras diferencias significativas en las características demográficas de los grupos (Tabla 1).

\section{Instrumentos}

INECO Frontal Screening (IFS): Es un instrumento breve de rastreo de las funciones de los lóbulos frontales que incorpora pruebas clásicas de funcionamiento ejecutivo. El punto de corte para sospecha de disfunción frontal es < 25 puntos en la puntuación total (IFSTOT), con sensibilidad del $96.2 \%$ y especificidad del $91.5 \%$. Tiene muy buena consistencia interna y se correlaciona 
Tabla 1. Características demográficas de un grupo de excombatientes del conflicto armado colombiano y un grupo control.

\begin{tabular}{|c|c|c|c|c|}
\hline & $\begin{array}{c}\text { Excombatientes } \\
\qquad \mathrm{N}=39\end{array}$ & $\begin{array}{c}\text { Control } \\
\mathrm{N}=14\end{array}$ & Estadístico & Valor $\mathrm{p}$ \\
\hline \multicolumn{5}{|l|}{ Sexo } \\
\hline Masculino, n (\%) & $34(87.2)$ & $10(71.4)$ & $X^{2}: 1,8 ; 1 \mathrm{gl}$ & 0.178 \\
\hline Femenino, n (\%) & $5(12.8)$ & $4(28.6)$ & & \\
\hline \multicolumn{5}{|l|}{ Edad } \\
\hline Media (DE) & $35(7.6)$ & $33(6.1)$ & 0.6 & 0.505 \\
\hline \multicolumn{5}{|l|}{ Escolaridad } \\
\hline Media (DE) & $10(2.8)$ & $10(2.7)$ & 0.3 & 0.717 \\
\hline \multicolumn{5}{|c|}{ Cociente intelectual } \\
\hline Media (DE) & 79 (10.1) & $85(10.4)$ & 2.0 & 0.044 \\
\hline
\end{tabular}

$N=$ Muestra; n: Frecuencia; DE: Desviación estándar.

significativamente con pruebas clásicas de funciones ejecutivas. Tiene las siguientes dimensiones: programación motora (PM), instrucciones en conflicto (IC), control motor inhibitorio $(\mathrm{CMI})$, dígitos atrás o en regresión (DA), memoria de trabajo verbal (MTV), memoria de trabajo espacial (MTE), capacidad de abstracción (CA), control inhibitorio verbal (CIV), índice de memoria de trabajo (IMT). Su administración toma unos 10 minutos, y otorga un perfil claro del funcionamiento ejecutivo de la persona evaluada, especialmente para sospecha de demencia fronto-temporal ${ }^{23}$.

Cuestionario de sensibilidad a la recompensa (SR) y sensibilidad al castigo (SC): evalúa el funcionamiento del SAC a través de la escala SR y el SIC a través de la escala $\mathrm{SC}^{24}$. Es autoadministrado y consta de 48 ítems, cada uno con respuesta dicotómica: sí/no, calificable con un punto a cada respuesta positiva. Los ítems impares evalúan SC, y los ítems pares valoran SR. La puntuación total de cada escala va de 0 a 24, y la media de las escalas oscila entre 10 y 12 , aproximadamente. En la escala SC, la media es de 11 puntos en ambos sexos, y en la escala SR, la media es 12 puntos (en hombres) y de 10 en mujeres. Ambas escalas han mostrado ser independientes. La consistencia interna de la escala SC es muy buena, alfa de Cronbach de $\alpha=0.83$ para hombres, $y \quad \alpha=0,82$ para mujeres; $y$ de la escala SR también, $\alpha=0,78$ para hombres, y $\alpha=0,75$ para mujeres. Las correlaciones test-retest oscilan entre 0.89 (escala SC) y 0.87 (escala SR) para un período de tres meses, y entre 0.57 (escala SC) y 0.61 (escala SR), para un período de tres años ${ }^{25}$.

\section{Análisis estadístico}

Los análisis se realizaron por medio del paquete estadístico SPSS versión 20. La descripción de las características de la muestra se realizó utilizando la frecuencia absoluta y porcentaje para sexo, y para las variables cuantitativas como edad, escolaridad, puntuaciones del Cuestionario de Sensibilidad al Castigo y a la recompensa y de $\mathrm{FE}$, se calculó la media y la desviación estándar (DE). Los grupos se compararon con la prueba Chi-Cuadrado para sexo y la U de Mann-Whitney para variables cuantitativas. Se calculó el tamaño del efecto con el valor $\mathrm{z}$ dividido por la raíz cuadrada de $\mathrm{n}^{26}$, y se consideró significativo cuando fue superior a 0.5 . Se calculó el coeficiente de correlación de Spearman para variables de escala, para establecer la correlación entre las variables del SCSR y las del INECO Frontal Screening.

\section{RESULTADOS}

En la Tabla 2 están las puntuaciones en el cuestionario de medición del SAC/SIC (SR-SC), y en la IFS para rastreo de $\mathrm{FE}$, del grupo de excombatientes y del grupo control. No hubo diferencias significativas entre los grupos en las escalas del cuestionario SR-SC. Se observó una puntuación total en el IFS significativamente inferior en el grupo de excombatientes. Esta puntuación inferior se explica por puntuaciones menores en las subpruebas de MTV, IC y CIV ( $p<0.05)$.

En la Tabla 3 se muestran las correlaciones entre el SAC/SIC (SR-SC) y las variables del IFS de ambos grupos. Se observó una correlación directa, relativamente alta $(r=0.65)$ y significativa en el grupo control entre la escala de SR y la CA del IFS. Se ven correlaciones directas moderadas $(0.40-0.45)$ y significativas en el grupo control entre la escala SR, la puntuación total del IFS, la MTV, la MTE y el CMI. Así mismo, entre la escala de SC y MTE. 
Tabla 2. Sistema de inhibición y activación conductual y función ejecutiva.

\begin{tabular}{lcccccc}
\hline & & $\begin{array}{c}\text { Excombatientes } \\
\text { Media (DE) }\end{array}$ & $\begin{array}{c}\text { Control } \\
\text { Media (DE) }\end{array}$ & D & *Estadístico & Valor $\mathbf{p}$ \\
\hline SCSR & & & & & & \\
& SC & $10.7(3.7)$ & $11.5(5.5)$ & 0.05 & 0.3 & 0.693 \\
& IFS & $11.6(4.1)$ & $10.4(4.1)$ & 0.11 & 0.8 & 0.400 \\
& & & & & \\
& PR & $2.6(0.7)$ & $2.9(0.2)$ & 0.21 & 1.6 & 0.103 \\
& IC & $2.5(0.7)$ & $3(0)$ & 0.30 & 2.3 & 0.020 \\
& CMI & $1.8(1.2)$ & $2.4(1.1)$ & 0.22 & 1.7 & 0.080 \\
& DA & $2.4(1.0)$ & $2.7(1.1)$ & 0.06 & 0.4 & 0.637 \\
& MTV & $0.8(0.8)$ & $1.7(0.5)$ & 0.46 & 3.5 & $<0.001$ \\
& MTE & $1.8(0.8)$ & $2.2(0.9)$ & 0.15 & 1.1 & 0.244 \\
& CA & $2.1(0.6)$ & $2.3(0.7)$ & 0.12 & 0.9 & 0.341 \\
& CIV & $3.8(2.2)$ & $5.2(0.9)$ & 0.25 & 1.9 & 0.048 \\
& IMT & $4.29(1.5)$ & $4.7(1.4)$ & 0.16 & 1.2 & 0.217 \\
& IFSTOT & $18.2(4.3)$ & $22.5(2.6)$ & 0.45 & 3.5 & $<0.001$ \\
\hline
\end{tabular}

*U de Mann-Whitney. D:Tamaño del efecto; DE: Desviación estándar; SC: Sensibilidad al castigo; SR: Sensibilidad a la recompensa; PM: Programación motora; IC: Instrucciones conflictivas; CMI: Control motor inhibitorio; DA: Dígitos atrás; MTV: Memoria de trabajo verbal; MTE: Memoria de trabajo espacial; CA: Capacidad de abstracción; CIV: Control inhibitorio verbal; IMT: Índice de memoria de trabajo; IFSTOT: Ineco puntuación total.

Tabla 3. Correlación entre sistema de inhibición y activación conductual y función ejecutiva.

\begin{tabular}{lcccc}
\hline & \multicolumn{2}{c}{ Excombatientes } & \multicolumn{2}{c}{ Control } \\
\hline SM & SC & SR & SC & SR \\
IC & -0.06 & 0.10 & -0.21 & 0.35 \\
CMI & 0.04 & 0.16 & 0 & 0 \\
DA & 0.00 & 0.06 & -0.02 & $0.40^{*}$ \\
MTV & -0.14 & -0.04 & -0.20 & -0.03 \\
MTE & -0.06 & -0.27 & 0.07 & $-0.42^{\star}$ \\
CA & -0.06 & -0.03 & $0.42^{\star}$ & $0.40^{*}$ \\
CIV & -0.32 & -0.26 & -0.25 & $0.65^{*}$ \\
IMT & 0.03 & -0.26 & -0.17 & 0.05 \\
IFSTOT & -0.08 & 0.02 & 0.08 & 0.22 \\
\hline
\end{tabular}

SC: Sensibilidad al castigo; SR: Sensibilidad a la recompensa; PM: Programación motora; IC: Instrucciones conflictivas; CMI: Control motor inhibitorio; DA: Dígitos atrás; MTV: Memoria de trabajo verbal; MTE: Memoria de trabajo espacial; CA: Capacidad de abstracción; CIV: Control inhibitorio verbal; IMT: Índice de memoria de trabajo; IFSTOT: Ineco puntuación total. * ${ }^{2}$ correlación es significativa a 0.05 . 


\section{DISCUSIÓN}

El principal hallazgo de esta investigación fue encontrar sospecha de disfunción ejecutiva en los excombatientes del conflicto armado colombiano, al comparar con un grupo similar de no combatientes. Esta disfunción se explica por una puntuación menor en la ejecución de IC, MTV y CIV de la prueba de rastreo IFS. Estos datos apoyan la hipótesis de una probable alteración de las funciones de los lóbulos frontales, especialmente relacionadas con el control inhibitorio, asociadas a los circuitos orbitofrontales, en personas con conductas violentas que tienen psicopatología y trastornos de personalidad antisocial, en diversos contextos ${ }^{27-29}$.

La exposición a combates en relación con guerras y otros tipos de conflictos armados genera alteraciones de la FE, explicadas por la presencia de estrés postraumático persistente, o por trauma de cráneo leve, o por la exposición corporal a ondas explosivas difusas, sin que se pueda documentar, en este último evento, la presencia de traumas específicos focales ${ }^{30-32}$.

De otro lado, similar a lo que se informa en la investigación actual, se afirma que la alteración de la función de los lóbulos frontales no se asocia con todas las dimensiones de la función ejecutiva, sino de manera específica con alteraciones del control inhibitorio, probablemente debido a disfunción de circuitos frontales orbitarios, que responderían de manera inadecuada a claves relacionadas con el contexto, lo tendría que ver con la presencia de estrés postraumático persistente ${ }^{33,34}$.

Algunos autores sugieren que las alteraciones neuropsicológicas inducidas por la exposición a situaciones de combates durante la guerra generan cambios en la memoria, por causa del mal funcionamiento de los circuitos temporales mediales, específicamente el hipocampo y el núcleo amigdalino, igualmente, producido por el estrés postraumático persistente ${ }^{35,36}$.

En la investigación que se presenta se encontró que las alteraciones en la puntuación total de la escala de FE (IFSTOT) dependía fundamentalmente de puntuaciones más bajas en dimensiones específicas, como la ejecución de IC, la MTV y el CIV. Las otras dimensiones de las funciones de los lóbulos frontales no mostraron diferencias significativas con los controles no combatientes. No se aplicaron pruebas de memoria controladas, para investigar si los excombatientes tenían otros problemas de memoria, más allá de los trastornos de memoria de trabajo verbal.

De otra parte, no se encontraron diferencias en los $\mathrm{SAC} / \mathrm{SIC}$ (SR-SC) entre los dos grupos de este estudio. Esto diverge de los reportes en los que se ha relacionado el SAC (SR) con trastornos externalizantes, las alteraciones del control de los impulsos y las conductas violentas ${ }^{3,5-7,37}$, que estarían ligadas a la participación en actos violentos como combatientes integrantes grupos armados irregulares. De igual forma, se descarta la presencia de problemas en el SIC (SC). Infortunadamente, no se indagó por la frecuencia del estrés postraumático ni de las alteraciones del estado de ánimo. Se ha informado que las alteraciones por $\mathrm{SC}$ se asocian con trastornos internalizantes, como los de ansiedad y los afectivos ${ }^{1,8,38,39}$.

El otro hallazgo importante de esta exploración fue la evidencia de correlaciones significativas y relativamente altas (0.65) entre el SAC (SR) y la CA, solo en el grupo de controles no combatientes; esto podría indicar que en los sujetos no expuestos a combates de esta muestra, la CA depende de la motivación relacionada con la recompensa. Esta correlación fue inversa, con coeficiente $r$ bajo $(-0.26)$ y no significativa, en el grupo de excombatientes.

Las correlaciones, en ambos grupos, para la SAC (SR) y el CMI, la MTV,la MTE y el IFSTOT, que fueron bajas y no significativas en el grupo de excombatientes, y las correlaciones moderadas entre el SIC (SC) y la MTE (r: 0,42 ) en el grupo de controles no combatientes, sugieren que la exposición a eventos traumáticos relacionados con combates en un conflicto irregular, como el caso del colombiano, rompen el enganche entre algunos elementos del funcionamiento ejecutivo.

Así mismo, se encontró que tanto en el grupo de excombatientes como en el de controles, el SIC que responde a estímulos temidos o aversivos, como el SAC relacionado con conducta impulsiva hacia lo placentero, que responde a las señales de recompensa y que se ha relacionado con características como la extroversión, la conducta sexual y la conducta agresiva ${ }^{1,2}$, están dentro de los parámetros normales. Estos hallazgos contrastan con un estudio que demostró que, tanto en la psicopatía primaria, como secundaria, los SAC/ SIC están alterados ${ }^{43}$.

De forma similar, otro estudio encontró que puntajes altos en el SAC explicaban las conductas agresivas e impulsivas en el trastorno antisocial de la personalidad ${ }^{44}$. Los datos del estudio exploratorio actual no corroboran la relación entre violencia y alteración del SAC/ SIC; sin embargo, la muestra es pequeña y no se indagó sobre psicopatología internalizante o externalizante, ni sobre trastornos de la personalidad.

Se ha relacionado el funcionamiento de los SAC/SIC (SR-SC) con la actividad de circuitos como el estriado ventral (núcleo accumbens), la amígdala y la corteza orbitofrontal $(\mathrm{COF})^{1,2,45}$, de neuronas monoaminégicas (dopamina, noradrenalina y serotonina), de los que depende el comportamiento motivacional relacionado con la activación y la inhibición conductuales.

Los datos reportados sugieren, en primera instancia, que los excombatientes del conflicto armado colombiano tienen un adecuado funcionamiento de estos circuitos rel- 
acionados con los SAC/SIC y los circuitos órbitofrontales de control inhibitorio, a pesar de que los excombatientes tienen alteraciones específicas en ejecución de IC y en CIV, relacionadas con el control inhibitorio cognitivo. La única variable de control inhibitorio que se correlacionó modestamente con SAC (SR) fue la de CMI.

Este es el primer estudio que explora las correlaciones entre variables neuropsicológicas del funcionamiento ejecutivo, usando una prueba muy breve de rastreo (IFS) y los SAC/SIC (SR-SC) en una muestra de sujetos expuestos a combate irregular. Lo que sugieren los datos es que la relación del SAC/SIC es con dimensiones específicas del funcionamiento ejecutivo, especialmente la CA, el CMI, la MTV y MTE.

La principal limitación de este estudio es el pequeño tamaño de la muestra, lo que impide hacer análisis multivariados, sobre todo, para analizar la influencia de la capacidad intelectual más baja en el grupo de excombatientes sobre el funcionamiento ejecutivo. A esta dificultad se suma la no exploración de otras funciones neuropsicológicas, como la memoria, que estaría alterada en personas expuestas a combates y explosiones. Una limitación adicional fue la ausencia de la evaluación de la muestra desde el punto de vista psicopatológico, ya que este aspecto influye tanto en los SAC/SIC como en las FE.

Para concluir, se encontraron alteraciones específicas del funcionamiento ejecutivo en excombatientes del con- flicto armado colombiano: en la ejecución de IC, MTV y CIV, que impactan sobre el funcionamiento ejecutivo global (IFSTOT). La actividad de los SAC/ SIC (SR$\mathrm{SC})$ fue normal. Los excombatientes presentan un rendimiento inferior en algunos dominios de las $\mathrm{FE}$, como la ejecución de IC, la MTV y el CIV, que impactarían en sus capacidades de anticipación y establecimiento de metas, diseño de planes e iniciación de conductas voluntarias y flexibilidad en el trabajo cognoscitivo, y el control inhibitorio, que podrían generar la toma de decisiones equivocadas ${ }^{21}$, en consonancia con múltiples estudios, que proponen a las disfunciones ejecutivas como determinantes importantes de conductas antisociales y violentas $^{46,47}$.

\section{DECLARACIÓN DE CONFLICTO DE INTERESES}

Los autores declaran no tener ningún conflicto de intereses.

Datos derivados del proyecto Procesamiento emocional y su modulación en personas reinsertadas del conflicto colombiano, pertenecientes a la Alta Consejería de la Presidencia de República, zona Antioquia. Financiado por Minciencias, contrato \#455-2009.

\section{REFERENCIAS}

1. Gray JA. Three fundamental emotion systems. Nat Emot Fundam Quest. 1994; 14:243-247.

2. Gray JA, McNaughton N. The neuropsychology of anxiety: An enquiry into the functions of the septohippocampal system. Oxford Univarsity Press, editor. New York; 2000.

3. Ganesh S, Kandasamy A, Sahayaraj US, Benegal V. Adult attention deficit hyperactivity disorder in patients with substance use disorders: A study from Southern India. Indian J Psychol Med. 2017; 39(1):59-62.

4. Ganesh S, Kandasamy A, Sahayaraj US, Benegal V. Behavioral activation and behavioral inhibition sensitivities in patients with substance use disorders: A study from India. Indian J Psychiatry. 2018; 60(3):346-50.

5. Gudiño OG, Nadeem E, Kataoka SH, Lau AS. Relative impact of violence exposure and immigrant stressors on Latino youth psychopathology. J Community Psychol. 2011; 39(3):316-35.

6. Gudiño OG, Nadeem E, Kataoka SH, Lau AS. Reinforcement sensitivity and risk for psychopathology following exposure to violence: A vulnerability-specificity model in Latino youth. Child Psychiatry Hum Dev. 2012; 43(2):306-21.

7. Watson $D$, Wiese $D$, Vaidya J, Tellegen A. The two general activation systems of affect: Structural findings, evolutionary considerations, and psychobiological evidence. J Pers Soc Psychol. 1999; 76(5):820-38.

8. Arfaie A, Safikhanlou S, Roodsari AB, Farnam A, Shafiee-Kandjani AR. Assessment of behavioral approach and behavioral inhibition systems in mood disorders. Basic Clin Neurosci. 2018; 9(4):261-68.

9. De Pascalis V, Sommer K, Scacchia P. Resting frontal asymmetry and reward sensitivity theory motivational traits. Sci Rep. 2018; 8(1):1-9.

10. Jones $E$, Gable PA. On the role of asymmetric frontal cortical activity in approach and withdrawal motivation: An updated review of the evidence on the role of asymmetric frontal cortical activity in approach and withdrawal motivation: An updated review of the evidence. Psychophysiology. 2018; 55(1):e12879. 
11. Krmpotich TD, Tregellas JR, Thompson LL, Banich MT, Klenk AM, Tanabe JL. Resting-state activity in the left executive control network is associated with behavioral approach and is increased in substance dependence. Drug Alcohol Depend. 2013; 129(1-2):1-7.

12. Johnson PL, Potts GF, Sánchez-Ramos J, Cimino CR. Self-reported impulsivity in Huntington's disease patients and relationship to executive dysfunction and reward responsiveness. J Clin Exp Neuropsychol. 2017;39(7): 694-706.

13. Giancola PR. Evidence for dorsolateral and orbital prefrontal cortical involvement in the expression of aggressive behavior. Aggress Behav. 1995; 21(6):431-50.

14. Filley CM, Price BH, Nell V, Antoinette T, Morgan AS, Bresnahan JF, et al. Toward an understanding of violence: Neurobehavioral aspects of unwarranted physical aggression: Aspen Neurobehavioral Conference consensus statement. Cogn Behav Neurol. 2001; 14(1):1-14.

15. Giancola PR. Executive functioning: A conceptual framework for alcohol-related aggression. Exp Clin Psychopharmacol. 2000; 8(4):576-97.

16. Verdejo-García A, Pérez-García M, Sánchez-Barrera M, Rodríguez-Fernández A, Gómez-Río M. Neuroimagen y drogodependencias: correlatos neuroanatómicos del consumo de cocaína, opiáceos, cannabis y éxtasis. Rev Neurol. 2007; 44(7):432-9.

17. Verdejo-García A, López-Torrecillas F, Giménez CO, Pérez-García M. Clinical implications and methodological challenges in the study of the neuropsychological correlates of cannabis, stimulant, and opioid abuse. Neuropsychol Rev. 2004; 14(1):1-41.

18. OPS. Informe mundial sobre la violencia y la salud. Organización Panamericana de la Salud; 2002.

19. Grande-García I. Neurociencia social: El maridaje entre la psicología social y las neurociencias cognitivas. Revisión e introducción a un nueva disciplina. An Psicol Psychol. 2009; 25(1):1-20.

20. Goldberg E. El cerebro ejecutivo: lóbulos frontales y mente civilizada. Planeta, Barcelona; 2015.

21. Tirapu-Ustárroz J, García-Molina A, Luna-Lario P, Roig-Rovira T, Pelegrín-Valero C. Modelos de funciones y control ejecutivo (II). Rev Neurol. 2008; 46(12):742-50.

22. Trujillo N, Pineda DA. Función ejecutiva en la investigación de los trastornos del comportamiento del niño y del adolescente. Rev Neuropsicol Neuropsiq Neurocien. 2008; 8(1):77-94.

23. Torralva T, Roca M, Gleichgerrecht E, López P, Manes F. INECO Frontal Screening (IFS): A brief, sensitive, and specific tool to assess executive functions in dementia. J Int Neuropsychol Soc. 2009; 15(5):777-86

24. Pinto MA. Sistemas de activación e inhibición conductual en los trastornos depresivos atendidos en la atención primaria en salud. Un estudio de seguimiento a 6 meses. Barcelona. UA de, editor. Victoria; 2004.

25. Torrubia R, Avila C, Moltó J, Caseras X. The sensitivity to punishment and sensitivity to reward questionnaire (SPSRQ) as a measure of Gray's anxiety and impulsivity dimensions. Pers Individ Dif. 2001; 31(6):837-62.

26. Fritz CO, Morris PE, Richler JJ. Effect size estimates: Current use, calculations, and interpretation. J Exp Psychol Genera. 2012; 141(1):2-18.

27. Blanchette I, Rutembesa E, Habimana E, Caparos S. Long-term cognitive correlates of exposure to trauma: Evidence from Rwanda. Psychol Trauma Theory. 2019; 11(2):147-55.

28. Kois LE, Blakey SM, Gardner BO, McNally MR, Johnson JL, Hamer RM, et al. Neuropsychological correlates of self-reported impulsivity and informant-reported maladaptive behaviour among veterans with posttraumatic stress disorder and traumatic brain injury history. Brain Inj. 2018; 32(12):1484-91.

29. Wallinius M, Nordholm J, Wagnström F, Billstedt E. Cognitive functioning and aggressive antisocial behaviors in young violent offenders. Psychiatry Res. 2019; 272:572-80.

30. Fogleman ND, Naaz F, Knight LK, Stoica T, Patton SC, Olson-Madden JH, et al. Reduced lateral prefrontal cortical volume is associated with performance on the modified lowa Gambling Task: A surface based morphometric analysis of previously deployed veterans. Psychiatry Res Neuroimaging. 2017; 267:1-8.

31. Janulewicz PA, Krengel MH, Maule A, White RF, Cirillo J, Sisson E, et al. Neuropsychological characteristics of Gulf War illness: A meta-analysis. Plos One. 2017; 12(5):e0177121.

32. Liverant GI, Amick MM, Black SK, Esterman M, Wisco BE, Gibian MC, et al. Associations among posttraumatic stress disorder symptoms, substance use, and affective attentional processing in OEF/OIF/OND Veterans. J Nerv Ment Dis. 2017; 205(9):725-31.

33. DeGutis J, Esterman M, McCulloch B, Rosenblatt A, Milberg W, McGlinchey R. Posttraumatic psychological symptoms are associated with reduced inhibitory control, not general executive dysfunction. J Int Neuropsychol Soc. 2015; 21(5):342-52.

34. Van Rooij SJ, Rademaker AR, Kennis M, Vink M, Kahn RS, Geuze E. Impaired right inferior frontal gyrus response to contextual cues in male veterans with PTSD during response inhibition. J Psychiatry Neurosci. 2014; 39(5):330-8.

35. Koso $M$, Hansen $S$. Executive function and memory in posttraumatic stress disorder: A study of Bosnian war veterans. Eur Psychiatry. 2006; 21(3):167-73.

36. Stricker NH, Lippa SM, Green DL, McGlynn SM, Grande LJ, Milberg WP, et al. Elevated rates of memory impairment in military service-members and veterans with posttraumatic stress disorder. J Clin Exp Neuropsychol. 2017; 39(8):768-85. 
37. Krueger RF, South SC. Externalizing disorders: Cluster 5 of the proposed meta-structure for DSM-V and ICD-11: Paper 6 of 7 of the thematic section: A proposal for a meta-structure for DSM-V and ICD-11. Psychol Med. 2009; 39(12):2061-70.

38. Johnson SL, Turner RJ, Iwata N. BIS/BAS levels and psychiatric disorder: An epidemiological study. J Psychopathol Behav Assess. 2003; 25(1):25-36.

39. Gable PA, Harmon-Jones E. Trait behavioral approach sensitivity (BAS) relates to early $(<150$ ms) electrocortical responses to appetitive stimuli. Soc Cogn Affect Neurosci. 2013; 8(7):795-8.

40. Kollmann B, Scholz V, Linke J, Kirsch P, Wessa M. Reward anticipation revisited-evidence from an fMRI study in euthymic bipolar I patients and healthy first-degree relatives. J Affect Disord. 2017; 219:178-86.

41. Sommer K, van der Molen MW, De Pascalis V. BIS/BAS sensitivity and emotional modulation in a prepulse-inhibition paradigm: A brain potential study. Physiol Behav. 2016; 154:100-13.

42. Li Y, Qiao L, Sun J, Wei D, Li W, Qiu J, et al. Gender-specific neuroanatomical basis of behavioral inhibition/approach systems (BIS/BAS) in a large sample of young adults: A voxel-based morphometric investigation. Behav Brain Res. 2014; 274:400-8.

43. Newman JP, MacCoon DG, Vaughn LJ, Sadeh N. Validating a distinction between primary and secondary psychopathy with measures of Gray's BIS and BAS constructs. J Abnorm Psychol. 2005; 114(2):319-23.

44. Quay HC. The psychobiology of undersocialized aggressive conduct disorder: A theoretical perspective. Dev Psychopathol. 1993; 5(1-2):165-80.

45. Rolls ET. On the brain and emotion. Behav Brain Sci. 2000; 23(2):219-28.

46. Raine A. Antisocial behavior and psychophysiology: A biosocial perspective and a prefrontal dysfunction hypothesis. Handbook of antisocial behavior. John Wiley \& Sons. New York; 1997. 289-304 p.

47. Morgan $A B$, Lilienfeld SO. A meta-analytic review of the relation between antisocial behavior and neuropsychological measures of executive function. Clin Psychol Rev. 2000; 20(1):113-36. 